\title{
THE INFLUENCE OF POLITICS ON THERAPEUTIC MODALITIES
}

Lilla HÁRDI, Cordelia Foundation for the Rehabilitation of Torture Victims, Balzac St. 37. 1136. Budapest ,Hungary. E-mail: hardi.lilla@cordelia.hu

\section{Cordelia Foundation for the Rehabilitation of Torture Victims}

has been actively working on the rehabilitation of torture survivor asylum seekers and refugees since 1996 as the only Hungarian trauma center in the global network of IRCT (International Rehabilitation and Research Council of Torture Victims). The center offers a multidimensional therapeutic help to seriously traumatized patients coming from different war zones of the world, like Somalia, Afghanistan, Iraq, Iran, etc.

Therapeutic methods: holistic, comprehesive, bio-psycho-social Rehabilitation must be, due to EU regulations available, appropriate and accessible at the earliest potential point after torture/trauma.

Our methods are verbal and non-verbal individual or group therapies.

Most of the patients come from different non-Christian societies, so we tailor the methods to their cultural background. The therapies are conducted through psychologically trained interpreters who achieve the proper interpreting skills in Cordelia. They also offer special information to us, the therapists on the proper culture.

As the therapeutic interventions used to form a circle of therapeutic modalities, the patients receive complex bio-psychosocial rehabilitation process from the first period of reaching the country.

Due to the recent changes of the political attitude during the past nearly 10 years we also had to change the therapuetic strategies. We do not have access to the tranzit zones, so we have only about sometimes less than 30 days to meet and to treat the seriously traumatized patients suffering of posttraumatic stress disorder, complex PTSD, major depression, anxiety issues, etc. They come and leave the country as soon as possible, only those remain who have no energy to continue their way to Europe for different reasons. Sometimes we can meet the patient only once.

\section{Sources}

1. Talmon, M. (1990). The Jossey-Bass social and behavioral science series. Single-session therapy: Maximizing the effect of the first (and often only) therapeutic encounter. San Francisco, CA, US: JosseyBass.

2. Hoyt, M.F. (1994). Constructive therapies. US, New York, The Guilford Press.

3. Evaristo Akerele \& Andriy Yuryev (2017): Single session psychotherapy forhumanitarian missions, International Journal of Mental Health

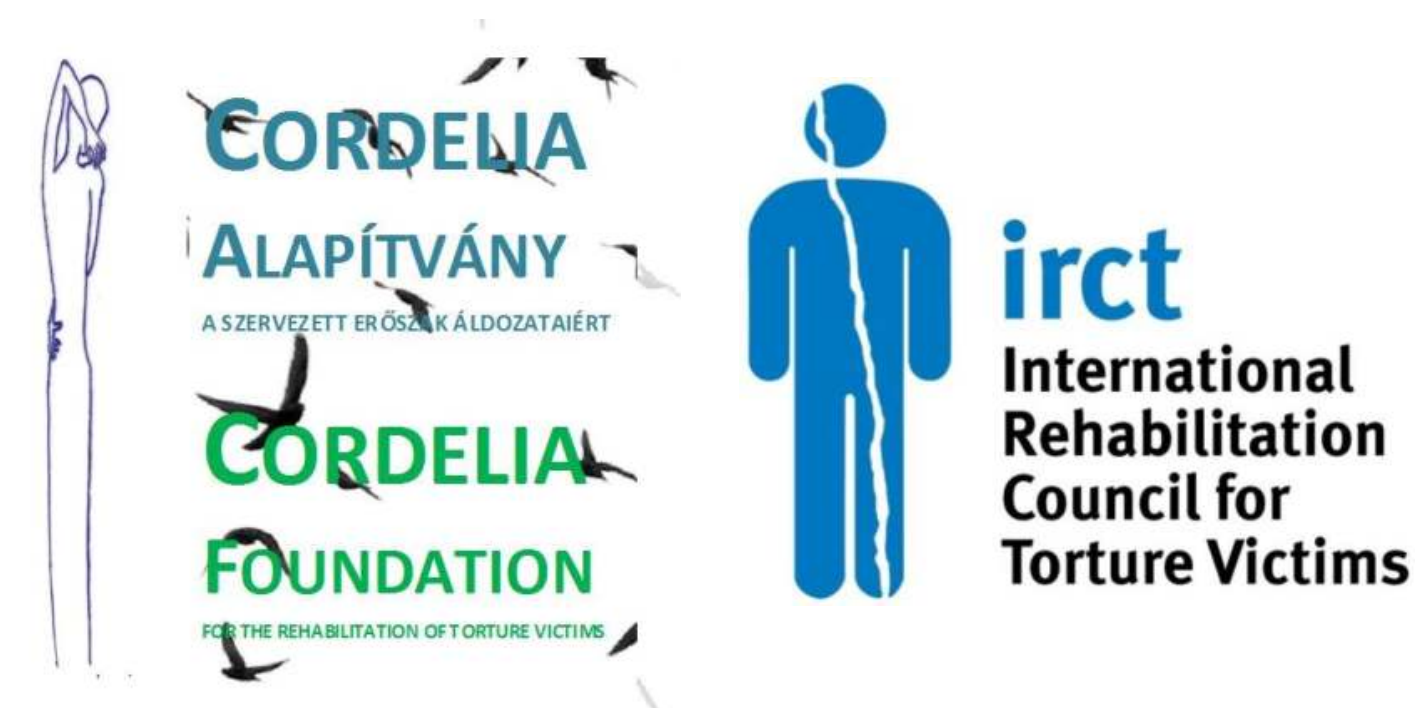

We began to adapt the Single Session Therapy methods to the need of this special group of patients.

1. Pre-session screening is not possible in the "classical meaning", so an immediate risk assessment (suicidality, acute psychotic symptoms, etc) takes place.

2. During the introduction (10-15 minutes) we explain our services and identify the main problems. We also try to evaluate the resilient capacity. Establish rapport.

3. The core session lasts for 60-80 minutes having supportive, cognitive and motivational elements like

a. building a trustful relationship with active listening and supportive responding

b. exploring the strengths, resources and evaluating the coping capacity

c. intervention (no history-taking ,focus on immediate concerns)

d. elaborating together on further perspectives

e. mobilising support

f. increasing self esteem

g. affirmation

h. encourage change

4. Risk assessment, minimising risk of retraumatization

5. Closing part (10-15 minutes): summarizing recommendations, referrals, encourage the use of acquired skills and knowledge.

Results: during period 01.06, 2019 our therapists met 108 patients.

Single session therapy was used with 26 patients.

Follow-up was not possible in most of the cases but we know from telephone calls and emails that many of them could use the acquired skills in finding their way in a new context.

Limitations: a regular follow-up is needed - with refugees it is not possible 